\title{
ON THE SCORE SEQUENCE \\ OF AN N-PARTITE TOURNAMENT
}

J. W. Moon

(received November 21, 1961)

Let the re be given $n(\geq 1)$ distinct sets of points $P_{i}=\left(P_{i 1}, \ldots, P_{\text {in }_{i}}\right)$, with $n_{i} \geq 1$, for $i=1, \ldots, n$. If joining each pair of points not in the same set is a line oriented towards one, and only one, point of the pair the resulting configuration will be called an n-partite tournament. If the line joining $P_{i j}$ and $P_{k} \ell$ is oriented towards the latter point we shall indicate this by $\mathrm{P}_{\mathrm{ij}} \rightarrow \mathrm{P}_{k}$, and similarly if the orientation is in the opposite sense.

By the score of $P_{i j}$ is meant the number, $v_{i j}$, of points $P_{k}$ such that $P_{i j} \rightarrow P_{k l}$ and by the score sequence of an $n$-partite tournament is meant the sets of scores $v_{i}=\left(v_{i 1}, \ldots, v_{i n_{i}}\right), i=1, \ldots, n$. With no Ioss of generality we may assume that $v_{i 1} \leq v_{i 2} \leq \cdots \leq v_{\text {in }_{i}}$ for all $i$. The object of this note is to give necessary and sufficient conditions for $n$ sets of integers to be the score sequence of an $\mathrm{n}$-partite tournament.

The se a re contained in

THEOREM 1. $n(\geq 1)$ sets of integers $v_{i}=\left(v_{i 1}, \ldots, v_{i n}\right)$, where $v_{i 1} \leq v_{i 2} \leq \cdots \leq v_{i n}$ and $n_{i} \geq 1$ for $i=1, \ldots, n$ form the score sequence of an n-partite tournament if, and only if,

Canad. Math. Bull. vol. 5, no. 1, Janua ry 1962. 
(1)

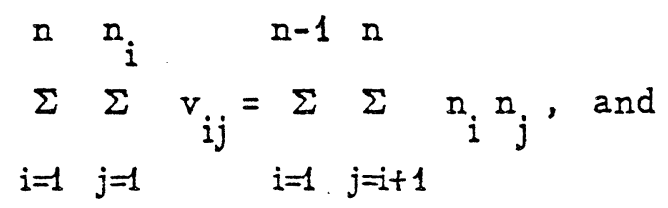

(2)

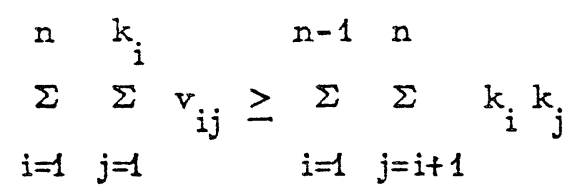

for all sets of integers $k_{i}, i=1, \ldots, n$, where $0 \leq k_{i} \leq n_{i}$, but not all $k_{i}=n_{i}$.

It is ea sily seen that (1) and (2) imply that n $0 \leq v_{i j} \leq\left(\sum_{s=1} n_{s}\right)-n_{i}$, for all $i$ and $j$ involved.

That the conditions are necessary is obvious since (1) simply requires that the sum of the scores be equal to the number of oriented lines in the configuration, and (2) states that the sum of the scores of the points in any proper subset of aII the points must be at least as large as the number of oriented lines joining points within this subset.

Before proceeding to the sufficiency part it will be convenient to state a few preliminary results.

If the points of an n-partite tournament can be separated into two mutually exclusive and exhaustive classes $A$ and $B$ such that each Iine which joins a point in $A$ to a point in $B$ is oriented towards the latter point then the configuration will be said to be reducible. To avoid making exceptions later the trivial 1 -partite tournament will be considered reducible, except when $n_{1}=1$. An $n$-partite toumament is irreducible if it is not reducible.

LEMMA 1. An n-partite tournament is irreducible if, and only if, its score sequence satisfies condition (2) with strict inequality holding throughout. 
This follows immediately from the definitions of the te rms involved.

An n-partite tournament is defined as being strongly connected if, and only if, for each ordered pair of distinct points, $P_{i j}$ and $P_{k l}$, there is a path from $P_{i j}$ to $P_{k \ell}$, i.e. a sequence of distinct points whose first element is $\mathbf{P}_{i j}$ ' whose last element is $\mathrm{P}_{\mathrm{k}}$, and such that from each point in the sequence issues a line oriented toward its successor in the sequence.

The following statement is similar to a theorem published by Roy [3] but the argument is somewhat different.

LEMMA 2. An n-partite tournament is strongly connected if, and only if, it is irreducible.

Necessity is obvious and if it is not strongly connected

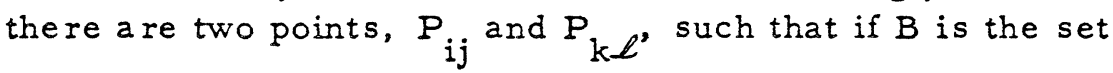
of all points which are reachable from $P_{i j}$ by some path, then

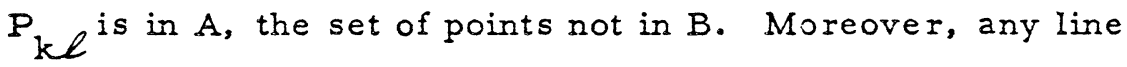
which joins a point in $A$ to a point in $B$ must be oriented towards the latter point, which implies reducibility by definition.

Straightforward considerations are sufficient to establish

LEMMA 3. Given sets of integers $V_{i}, i=1, \ldots, n$, satisfying the conditions of Theorem 1 , for which there exist integers $h_{i}, 0 \leq h_{i} \leq n_{i}$, for $i=1, \ldots$ n with not all $h_{i}=n_{i}$, such that equality holds in (2) when $k_{i}=h_{i}$. Then the sets $v_{i}^{\prime}=\left(v_{i 1}, \ldots, v_{i h_{i}}\right)$ and the sets $v_{i}^{\prime \prime}=\left(v_{i h_{i}+1}-b_{i}, \ldots, v_{i n_{i}}-b_{i}\right)$, n where $b_{i}=\left(\sum_{s=1}^{\Sigma} h_{s}\right)-h_{i}$, satisfy the conditions of Theorem 1 with $n_{i}$ replaced by $h_{i}$ and $n_{i}-h_{i}$, respectively, for $i=1, \ldots, n$. 
Hence if the re exist two $n$-partite toumaments whose score sequences are the $V_{i}^{\prime}$ 's and $V_{i}{ }^{\prime \prime}, s$, respectively, then combining them in a rather obvious fashion yields an $n$-partite tournament whose score sequence consists of the original sets $v_{i}, i=1, \ldots, n$. The inte rpretation is clear whenever any $h_{i}=0$ or $n_{i}$.

The proof of the sufficiency part of Theorem 1 now proceeds by multiple induction, first over the number of sets of points involved.

The case $\mathrm{n}=1$ is trivial, so we now assume the theorem has been established for up to $n-1$ sets of points, for some n $>1$.

Suppose we have $n$ sets, $V_{i}$, of integers, satisfying the conditions of Theorem 1 in which $v_{n 1}=\ldots=v_{n n}=0$. Then by our induction hypothesis and Lemma 3 , with $h_{1}=\ldots=h_{n-1}=0$, and $h_{n}=n$, we may assert the existence of an $n$-partite tournament whose score sequence consists of the $V_{i}^{\prime}$.

For the second induction assume the required result has been proved for all cases where $0 \leq v_{n n} \leq t-1$ and consider $\mathrm{n}$ sets, $\mathrm{V}_{i}$, of integers, satisfying the conditions of Theorem 1 with $\mathrm{v}_{n n_{n}}=t$ with strict inequality holding throughout in (2).

Our third induction is now upon $\mathcal{L}$, the number of scores in $\mathrm{V}_{\mathrm{n}}$ equal to $\mathrm{t}$.

If $l=1$ we modify the given sets, $V_{i}$, by subtracting one from $v_{n n}$, which is $\geq 1$ by hypothesis, and adding one to, say, the smallest $v_{i j}$, where $i<n$, in such a way as to leave all the numbers in each set still arranged in non-decrea sing order. That is, if say, $\left(v_{11}, v_{12}, v_{13}\right)=(0,0,1)$, the one would not 
be added to $\mathrm{v}_{11}$ but to $\mathrm{v}_{12}$. This change does not effect the total sum of the numbers, so (1) is still satisfied. Also any of the sums in (2) are decreased by at most one but as we had strict inequality holding throughout originally, by assumption, we see that the modified sets of numbers satisfy (2).

The maximum score in $\mathrm{V}_{\mathrm{n}}$ is now $\mathrm{t}-1$, so by our induction hypothesis, for the second induction, there exists an $n$-partite tournament whose score sequence is the set obtained by modifying the $\mathrm{V}_{i}^{\prime} \mathrm{s}$. We now need to change this $n$-partite tournament to one whose score sequence consists of the unmodified $V_{i}^{\prime}$ s.

Let $P_{i j}$ be the point whose score was increased by one.

Two cases now present themselves in the n-partite tournament whose score sequence consists of the modified set of $V_{i}^{\prime} s$. If $P_{i j} \rightarrow P_{n n}$ then by simply reversing the orientation of the line joining $P_{i j}$ and $P_{n n_{n}}$ we obtain a configuration whose score sequence is the original set of $\mathrm{V}_{i}^{\prime}$ s. When, however, $P_{n n} \rightarrow P_{i j}$ another approach is required.

If the $n$-partite tournament assured us by the induction hypothesis is reducible then $P_{n n_{n}}$ must be in the set labelled $B$ in the definition of reducibility. This follows from Lemma 1 and the fact that any partial sum of the modified set of $V_{i}^{\prime} s$ giving equality in (2) must have included the score of $P_{n n} \cdot P_{i j}$ must also be in $B$ since $P_{n n_{n}} \rightarrow P_{i j}$. But this is impossible using Lemma 1 again, since any partial sum, in (2), of the modified set of $\mathrm{V}_{i}^{\prime}$ 's which included the scores of both $P_{i j}$ and $P_{n n}$ was unchanged from what it was originally; hence strict inequality would hold and the only alternative, under our 
assumptions, is that if $P_{n n_{n}} \rightarrow P_{i j}$ then the configuration is irreducible.

By Lemma 2 there exists a path from $P_{i j}$ to $P_{n n}$.

The only effect upon the scores caused by reversing the orientation of all the lines in this path will be to decrease $P_{i j}$ 's by one and to increase $P_{n n}$ 's by one.

So in either case we have produced an n-partite tournament whose score sequence consists of the original set of $\mathrm{V}_{i}^{\prime} \mathrm{s}$.

We next drop the assumption that strict inequality held throughout in (2). What has already been shown along with application of Lemma 3, repeated if necessary, completes the proof for the case $\mathscr{L}=1$.

Now as sume the assertion has been demonstrated for $v_{n n}=t$ and up to $\ell=r-1 \geq 1$. Again we consider sets of numbers $V_{i}, i=1, \ldots, n$, in which the last $r$ elements of $v_{n}$ are equal to $t$, and which satisfy the conditions of Theorem 1 with strict inequality holding throughout in (2). The same scheme, only this time reducing $V_{n n_{n}}-(r-1)$ by one, suffices to complete the proof of the third induction.

But this completes also the proof of the induction on the maximum number in the nth set of numbers, which demonstrates that the theorem is valid for $n$ sets of numbers, which completes the argument for the pruof of the original statement, by induction.

$$
\text { COROLLARY 1. A set of integers } u_{1} \leq u_{2} \leq \cdots \leq u_{n}
$$
is the score sequence of an ordinary round-robin tournament if, and only if,

(3) $\sum_{i=1}^{k} u_{i} \geq\left(\begin{array}{l}k \\ 2\end{array}\right)$ for $k=1,2, \ldots, n$ with equality holding when $k=n$. 
This was first proved by Landau [2], without using the concept of irreducibility, and follows immediately from Theorem 1 upon setting $n_{1}=n_{2}=\ldots=n_{n}=1$.

Gale [1] and Ryser [4] have given necessary and sufficient conditions for the existence of a matrix of $0^{\prime} s$ and $1^{\prime} s$ with prescribed row and column sums. It can be shown that the following statement is equivalent to their theorem.

COROLLARY 2. There exists an $m \times n$ matrix of $0^{\prime} s$ and $1^{\prime} \mathrm{s}$ with row sums $r_{i}$, where $r_{1} \leq r_{2} \leq \ldots \leq r_{m}$ and column sums $c_{j}$, where $c_{1} \geq c_{2} \geq \cdots \geq c_{n}$ if, and only if,

$$
\sum_{i=1}^{k} r_{i}+\sum_{j=1}^{\ell}\left(m-c_{j}\right) \geq k l,
$$

for $k=0, \ldots, n$ and $l=0, \ldots, n$, with equality holding when $\mathrm{k}=\mathrm{m}$ and $\mathscr{L}=\mathrm{n}$.

The proof of this involves applying Theorem 1 to a ssert the equivalence of the hypothesis and the existence of a bipartite tournament with score sequence $v_{1}=\left(r_{1}, \ldots, r_{m}\right)$ and $v_{2}=\left(m-c_{1}, \ldots, m-c_{n}\right)$ and constructing the required $m$ x n matrix, $M=\left\|a_{i j}\right\|$, where $a_{i j}$ is 1 , or 0 , according as $P_{1 i} \rightarrow P_{2 j}$ or $P_{2 j} \rightarrow P_{1 i}$.

\section{REFERENCES}

1. D. Gale, "A theorem on flows in networks," Pac. J. Math., 7 (1957), 1073-1082.

2. H.G. Landau, "On dominance relations and the structure of animal societies: III. The condition for a score structure," BuIl. Math. Biophysics, 15(1953), 143-148. 
3. B. Roy, "Sur quelques proprietés des graphes fortement connexes," C. R. Acad. Sci. Paris, 249 (1959), 21512152.

4. H.J. Ryser, "Combinatorial properties of matrices of $0^{\prime}$ 's and 1's, "Can. J. Math. 9 (1957), 371-377.

University of Alberta 